\title{
Zur Aufgabe des christlichen Kommunikators: Theologische Überlegungen
}

\author{
von Patrick A. Kalilombe
}

Es geht mir hier darum, einige Gedanken zu äußern, wie wir als Christen unsere Aufgabe als Kommunikatoren sehen können, welche Bedeutung sie hat und welche Herausforderung sie für jene bedeutet, die aktiv im Kommunikationswesen engagiert sind.

Ich bin der Ansicht, daß Kommunikatoren zunächst einmal Kommunikatoren sind und dann erst Christen. Die zwei Komponenten sind beim christlichen Kommunikator in einer Person vereint. Schon allein die Tatsache, Kommunikator zu sein, bedeutet eine sehr hohe Aufgabe. Darüber hinaus aber auch noch Christ zu sein besagt, daß die Aufgabe als Kommunikator von christlichem Ideengut erfüllt und belebt wird. Dies ist von besonderer Bedeutung, denn nicht jeder Kommunikator geht seiner Aufgabe unter christlichen Aspekten nach.

Es ist schon eine sehr hohe Aufgabe, Kommunikator zu sein. Der Kommunikator leistet einen Dienst im Mittelpunkt des menschlichen Seins. Der Mensch ist durch die Schöpfung und damit durch Gottes Plan ein soziales Wesen. Auch die Philosophen sagen uns das, und wir fragen uns, was sie eigentlich damit meinen. Gott hat den Menschen so geschaffen, daß er nicht existieren und sich verwirklichen kann, wenn er nicht in Verbindung mit anderen steht, in einer sinnvollen, wirksamen und freien Verbindung. Der Mensch wurde so geschaffen, daß er seiner Bestimmung nicht voll gerecht werden kann, wenn er nicht in Kontakt mit anderen Menschen steht. Schon unsere physische Natur, die Art und Weise, wie wir geboren werden und heranwachsen, zeigt, daß wir nach Gottes Willen in Gesellschaft leben. Wir sind nicht wie die übrigen Lebewesen, die gleich nach der Geburt auf eigenen Füßen stehen. Wir sind auf menschliche Wesen angewiesen, auf die wechselseitige Wirkung menschlicher Beziehungen, auf das Zusammenspiel von Diensten, die angeboten und angenommen werden, damit wir von Geburt an zu wirklichen menschlichen Wesen heranwachsen. Wir können unsere Aufgabe als menschliche Wesen nur in der Gemeinschaft verwirklichen, wenn wir in einer bestimmten Beziehung zu anderen stehen, die gegenwärtig, sinnvoll und gültig wird, soweit sie aktive Kommunikation ist. Dies ist eine freie Kommunikation, wirksam in dem Sinne, daß sie tatsächlich auf die andere Person zielt, denn der Mensch neigt dazu, sich auf sich selbst zu konzentrieren und sich abzukapseln. Geschieht dies, dann gibt es keine Kommunikation, und der Mensch ist keine Person. Wenn ich jedoch mein Wesen, meine Person, das, was mich zu dem macht, was ich bin, mitteilen kann, dann lasse ich andere daran teilhaben, und der andere kann das gleiche tun, indem er sein eigenes Wesen, den kostbarsten Teil seiner

Bischof Patrick A. Kalilombe (42) von Lilongwe, Malawi, gilt von der Exegese kommend als einer der führenden Theologen des heutigen Afrika. Bei der Konferenz der christlichen Kommunikationsverantwortlichen Ost- und Zentralafrikas vom 25. bis 30 . August 1975 in Blantyre (vgl. in dieser Ausgabe S. 41) hielt er das Eröffnungsreferat, welches hier in einer bearbeiteten Fassung als afrikanischer Beitrag zu einer Theologie der Kommunikation veröfentlicht wird. 
selbst, mit mir teilt. Dies ist nicht sein Äußeres, sondern das, was er sich wirklich selbst bedeutet. Wenn er mich daran teilhaben läßt, wächst zwischen uns eine persönliche Beziehung. Ich bin nicht mehr allein, mein Menschsein wird vielmehr sinnvoller, da ich jetzt in menschlicher Gemeinschaft mit anderen bin.

Daher umfaßt diese menschliche Kommunikation in erster Linie Information. Die Menschen müssen um meine Zweifel, meine Ansichten, meine Gefühle, meine Pläne, meine Reaktionen, meine Handlung wissen, sie müssen wissen, wie ich zu Realitäten stehe, was ich vorhabe, müssen um den Erfolg oder Mißerfolg meiner Pläne wissen. Was ich für die Zukunft plane, was ich als Mensch empfinde, wenn ich um mich blicke und sehe, daß ich ein Stück und Teil dieser Welt bin. Das ist es, was uns wirklich zu dem macht, was wir sind.

Menschliche Kommunikation bedeutet, dieses Wesen meiner selbst mit dem anderen zu teilen, und sie bedarf dazu bestimmter Mittel, die es mir erlauben, aus mir herauszugehen, und dem anderen die Möglichkeit geben, auf dies "Geschenk meiner selbst" zu reagieren, indem er mich ebenfalls an sich teilhaben läßt. Durch diese Kommunikation werden wir zu Personen. Gottes Absicht, uns zu sozialen Wesen zu machen, ließ ihn für den Menschen eine ganz besondere Welt bauen. Gott dachte sich diese Welt, die Welt des Menschen, als eine große Familie, in der jeder nicht für sich selbst leben sollte, sondern für den anderen. Er ist in der Tat ein Einzelwesen, ein in sich geschlossenes, wertvolles Einzelwesen, aber sein Wert ist abhängig davon, was er für andere ist. Je mehr einer liebt, desto mehr erfüllt er seine Funktion vor Gott, denn Gott hat den Menschen dazu gerufen, in der Gesellschaft anderer zu leben und seine Familie in dieser Gesellschaft aufzubauen, die Welt aufzubauen, seine Welt fortzuführen. Gott schuf die Welt auf ganz besondere Weise. Er hat sie nicht vollendet. Er wollte, daß sie ein Paradies würde. Er wollte, daß die Welt eine Art Szenerie darstellt, vor der die Menschen als Ebenbilder seiner selbst das verwirl:lichen, was in Gott geschieht, sein Leben in der Dreifaltigkeit, die eine Wesensmitteilung der drei Personen und so reich ist, daß wir deswegen von Personen sprechen. Das soll auf menschlicher Ebene in der Welt nachvollzogen werden. Und wir vollziehen es nach, wenn unsere gegenseitige Beziehung so ist, daß wir zusammen füreinander kreativ sein können, nicht nur kreativ für einzelne, sondern so, daß die ganze Menschheit besser wird. Wenn also der Mensch so lebt, daß er die Welt besser macht, als er sie vorgefunden hat, erfüllt er seine erste Pflicht, die er als Mensch hat, und diese Gestaltung für ein besseres Leben, zu einem besseren Abbild von Gottes eigenem "Haus“, kann nur in der Gemeinschaft erfolgen. Dafür gab Gott uns die Sprache. Die Sprache, die Kommunikation, die Möglichkeit, mit anderen in Verbindung zu sein, heißt nicht nur, anderen unsere Gefühle, unser Wissen, unsere Information mitzuteilen, und sie daran teilhaben zu lassen, sondern auch, daß wir Partner und Mitarbeiter werden bei unserer gemeinsamen Aufgabe der Vollendung dieser Welt nach dem Willen Gottes. Dies ist das eigentliche Ziel der Kommunikation. Die Menschen sollen eins sein, sie sollen einander kennen, sollen füreinander offen sein und zusammenarbeiten für das Kommen des Reiches Gottes in dieser Welt. So ist es letztlich der Aufbau des Gottesreiches, der die Kommunikation erfordert. Menschliche Kommunikation ist ein gegenseitiges Teilhaben-lassen an den Möglichkeiten des Menschen, um das Reich zu errichten. Deswegen teilen wir nicht nur unsere Kenntnisse einander mit, sondern auch unsere Gefühle, Ansichten, Pläne, unsere Weltanschauung. Deshalb ist Kommunikation sehr heilig. Erst durch sie wird nämlich der Mensch zu dem, was er nach Gottes Willen sein soll. 
Ich glaube, es ist gut für Kommunikatoren, diese Bedeutung klar zu erkennen, denn gerade sie sind aufgefordert, die notwendige Kommunikation des Menschen zu fördern. Bevor wir zu Massenkommunikatoren oder modernen Kommunikatoren mit all ihren hochentwickelten Mitteln wurden, hat schon von Anfang an eine Kommunikation bestanden. Das zeigt schon das erste Kapitel der Genesis, da Gott den Menschen erschafft. Der Autor des ersten Kapitels sagt: Und Gott schuf den Menschen nach seinem Ebenbild. Er fügt hinzu: Er schuf sie als Mann und Frau. Was bedeutet dies? Es heißt, daß er die Menschen verschieden schuf, so daß kein einzelner Mensch oder keine einzelne Gruppe menschlicher Wesen ihren Auftrag als Menschen auf dieser Welt allein erfüllen kann. Nur durch das gegenseitige Sich-Ergänzen, durch Geben und Empfangen und Miteinander-Teilen gelingt es, die Welt aufzubauen. Das ist es, was der alte Schreiber sagt: Er schuf sie nach seinem Ebenbild. Als Mann und Frau schuf er sie und gab ihnen den Auftrag: Geht nun hin und baut diese Welt auf!

Kommunikation erwächst aus der Tatsache, daß Gott die Errichtung seiner Welt auf der Zusammenarbeit, auf der gegenseitigen Ergänzung, gegründet hat. Kein einzelner ist imstande, die Aufgabe des Menschen allein zu erfüllen, keine einzelne Gesellschaft, nicht einmal eine Kultur, ein Land, eine Nation kann allein der menschlichen Bestimmung gerecht werden. Nicht einmal einem Zeitalter, einer Periode der menschlichen Geschichte gelingt es, die Bestimmung des Menschen voll zum Ausdruck zu bringen. Nur durch ein fortwährendes Geben und Mitteilen, Teilen und Zusammenlegen der einzelnen Charismen von Gottes Weisheit und Gottes Macht, an denen er uns ständig teilhaben läßt, erreicht der Mensch langsam seine Bestimmung, wird er zum Ebenbild Gottes. Kein einzelner Mensch vermag das zu tun, was die Welt nach Gottes Wunsch tun soll. Wenn jedoch der Mensch weitergibt, was er empfangen hat, und er den anderen bittet, ebenfalls das zu geben, was er von Gott empfangen hat, und sie alle diese Gaben zusammenlegen und versuchen, sie zu einem sinnvollen Ganzen zu vereinen, dann können sie Gottes Absichten mit dieser Welt erfüllen. Kommunikation bedeutet, das gegenseitige Sich-Ergänzen jener Gaben zu ermöglichen, die wir alle empfangen haben. Kommunikation hilft uns, sie zusammenzufügen zu einem sinnvollen Ganzen, so daß sie uns allen helfen können, in der Zusammenarbeit die menschliche Bestimmung zu erfüllen. Sie ist daher eine heilige Pflicht.

Es gab auch schon Kommunikation, bevor die mächtigen modernen Medien entstanden. Aber heute liegt die besondere Verantwortung der Kommunikatoren darin, daß sie sehr mächtige und wirksame Mittel in Händen haben, nicht nur um diese Kommunikation unter den Menschen zu ermöglichen, sondern auch um sie mächtiger, wirksamer und sinnvoller zu machen. Durch die Entwicklung der Kommunikationsmittel in technischer wie auch sozialer und psychologischer Hinsicht, aber auch durch die Entwicklung der Verständigungs- und Kommunikationsmöglichkeiten selbst mit ihren Techniken und Mechanismen können die Menschen heute die Kommunikation noch stärker intensivieren und ihrer ursprünglichen Kraft noch mehr Effizienz geben. Die Menschen haben immer Kommunikation betrieben, aber heute ist diese Kommunikation wegen der Entwicklung der Kommunikationswissenschaften und -techniken viel einflußreicher. In ihr stecken größere Möglichkeiten, aber auch eine Herausforderung an die modernen Kommunikatoren. Sie haben eine größere Macht als in der Vergangenheit, die Welt aufzubauen oder zu zerstören.

Das Bild der Schrift von den Kommunikatoren ist ein zweifaches. Eines ist das vom Turm zu Babel im 11. Kapitel der Genesis. Das andere ist das Pfingsterlebnis im 
zweiten Kapitel der Apostelgeschichte. Beide stellen die Kommunikatoren dar. In Babel versuchen die Menschen, ihre eigene Welt zu errichten, die sich von jener, die Gott gewollt hat, unterscheidet. Sie wollen ihren eigenen Himmel bauen, wollen dorthin gelangen, wo Gott ist und neben Gott sitzen, sie wollen selbst Gott sein. Da diese Sprache, Sprache der Rebellion, Sprache des Kampfes gegen Gott ist, reißt sie die Menschen auseinander. Sie wird nicht kommunikativ, weil sie jeden Menschen in sein eigenes Gehäuse einschließt. Immer wenn die Menschen Gott nicht als Mittelpunkt der wahren Kommunikation akzeptieren, als den, von dem die Kommunikation ausgeht, werden sie uneins und entwickeln verschiedene Sprachen; denn die Sprache, welche echte Kommunikation erst möglich und wirksam macht, ist nichts anderes als die Liebe: Das Annehmen des Menschen als Bruder. Dies ist die Grundlage der Kommunikation im Blick auf die Zusammenarbeit beim Aufbau der Welt. Wenn wir uns nicht als Brüder fühlten, bestünde kein Grund, sich zusammenzuschließen, um zusammen zu arbeiten am Aufbau der einen Welt. Niemals wird man wirklich bereit sein, das zusammenzulegen, was man besitzt, um sich gegenseitig zu ergänzen, wenn man sich nicht als Glied derselben Familie, als Teil derselben Welt fühlt, denn sonst ist jeder für sich eine Insel. Wenn wir nicht bereit sind, alle Kinder desselben Gottes zu sein, denen dieselbe Aufgabe und dasselbe Schicksal gegeben ist, haben wir keinen Grund, uns als Brüder zu fühlen. Wenn wir nicht vom gleichen Vater und derselben Mutter abstammen, sind wir mit den anderen nicht verwandt. Wenn die Menschen vergessen, daß sie Kinder Gottes und Brüder sind, wird die Kommunikation unterbrochen. Es mag zwar wie Kommunikation aussehen, aber es handelt sich um eine falsche, nicht authentische Kommunikation. Sie schließt jeden einzelnen in sein eigenes Gehäuse ein, so daß kein Fortschritt, keine Entwicklung auf das Reich Gottes hin mehr möglich ist.

Anders sieht es im zweiten Kapitel der Apostelgeschichte aus. Wir sehen, wie der Geist Gottes, der von oben kommt, die Menschen vereint vorfindet, physisch vereint in Jerusalem, wiewohl sie aus allen möglichen Ländern stammen, verschiedene Sprachen sprechen, verschiedenen Kulturbereichen angehören usw. Wenn die vom Heiligen Geist erfüllten Apostel zu ihnen kommen, wird ihnen bewußt, daß sie alle dieselbe Sprache sprechen. In Wirklichkeit reden sie nicht alle in derselben Sprache. Jeder spricht seine Muttersprache. Aber sie verstehen einander. Tatsächlich sind ihre Sprachen alle Dialekte der einen Hauptsprache, der menschlichen Sprache. Daher können sie auch alle die Apostel verstehen, wenn diese sprechen. Und sie waren erstaunt und fragten: Sind nicht alle Galiläer? Wie ist es möglich, daß wir sie alle in unserer Muttersprache verstehen, wenn sie galiläisch sprechen? Der Grund war einfach: Sie sprachen die Sprache des Geistes. Die Sprache des Geistes ist die der Einheit, der Liebe, der Erfüllung des Willens Gottes: Sie verbindet. Sie reißt die Menschen nicht auseinander wie der Turm von Babel, sondern verbindet sie. Daher müssen die Kommunikatoren unserer Zeit vom Heiligen Geist erfüllt sein. Dann werden sie imstande sein, eine Sprache zu sprechen, die die Menschen vereint und sie tatsächlich befähigt, das Reich Gottes zu verwirklichen.

Noch ein Gedanke: Mit seinem Dienst als Kommunikator unterstützt der Christ auf allen Gebieten, auf denen er tätig ist, sei es durch Zeitschriften, Zeitungen oder Bücher, durch Hörfunk und AV-Medien, den Kommunikator schlechthin, nämlich Christus. Tatsächlich ist Christus allein der wirkliche und wahre Kommunikator, derjenige, der weiß, wie man Menschen eint. Kommunikation bedeutet die Offenbarung dessen, was jemand von anderen erfahren möchte, nämlich Selbst-Offenbarung, so 
daß die beiden Offenbarungen helfen zur Zusammenarbeit bei der gemeinsamen Aufgabe für das Kommen des Reiches Gottes. Dies hat Christus selbst schlechthin getan. Er ist personifizierte Kommunikation ${ }^{1}$. Er ist derjenige, der Gott unter uns gegenwärtig machte. Er offenbarte Gott. Wir wußten nicht, was Gott war, wer er wirklich war. Und wir hätten ihn gerne gesehen, hätten gerne Gott geschaut. Selbst wenn es uns einer erklärt hätte, hätten wir nicht ganz verstanden, wer Gott war, bis Gott selbst offenbar wurde im Fleisch. Er wurde Mensch und stand so zu unserer Verfügung. Er offenbarte sich in unserer Alltäglichkeit. Er wurde so sehr wie wir, daß wir sagen konnten: Jetzt sehe ich, wer Gott ist, wie er mit uns ist, in welcher Beziehung er zu uns steht. So ist Gott. Das ist Gott. So wurde der unsichtbare Gott sichtbar in Christi Menschsein, wie es sich in seinem Verwandtschaftsverhältnis zu uns offenbart. Nun lernen wir in Christus Gott kennen. Gott offenbarte sich in Christus. In Christus sehen wir aber auch das vollkommene Ebenbild des Menschen, den wahren Menschen, wie Gott ihn wollte. Seit wir Christus sehen, der unter uns Menschen lebt, sehen wir einen authentischen, vollblütigen Menschen, alles, aber auch alles, was wirklich menschlich ist, wurde in ihm offenbar. Alles, ausgenommen die Sünde, und die Sünde ist nicht menschlich im Sinne von Gottes ursprünglichem Plan.

Sie ist eine Schöpfung des Menschen, ein Ungeheuer. Alles, was wirklich menschlich ist, wurde in Christus offenbar. So wurde Christus für uns auch: Kommunikation. Er zeigt uns, wer wir sind, wer wir sein sollten, wer wir zu sein haben, indem er uns Gott brachte, den wirklichen und offenbaren Gott, nicht den Gott da oben, mit dem wir nichts zu tun haben. Im alten Chichewa-Katechismus pflegten wir zu sagen: Gott ist Geist. Das klingt alles sehr schön. Er soll Geist sein, was soll's? Wir haben kein Interesse an einem reinen Geistwesen. Wir wollen einen Gott, der mit uns in Verbindung steht, der für uns in unserem Leben Bedeutung hat. Christus wurde dieser Gott, mit einer vollen Bedeutung für uns menschliche Wesen. Er hat sich mit uns verbunden und uns gezeigt, wer wir sind in unserer Beziehung zu Gott und zueinander. So war Christus wirkliche Offenbarung, wirkliche Kommunikation. Er brachte uns in eine Beziehung zu Gott und zueinander. In seinem Wirken ist die Aufgabe des Kommunikators zusammengefaßt. Er sagte: Ich gebe euch ein Gebot: Liebet einander. An dem Tage, an dem ihr Gott liebt und einander liebt, habt ihr das erfüllt, wofür ich kämpfe. Tatsächlich bedeutet die Tatsache seines Kommunikator-Seins, daß er in der ganzen Welt die Kraft seiner Liebe unter den Menschen verbreitete, denn das ist die Kraft, die die Welt aufbaut.

Als Kommunikatoren nehmen wir an dieser Aufgabe teil. Wir versuchen, seine Aufgabe der Versöhnung fortzuführen, es den Menschen leichter zu machen, sich gegenseitig zu kennen, sich selbst vor allem dem anderen wirklich zu offenbaren im Blick auf das gemeinsame Werk der Erfüllung des Planes Gottes von seinem Reich. So führen wir heute in unserer modernen Umwelt die Aufgabe Christi als Kommunikator fort. Dies ist eine sehr hohe Aufgabe. Auch wer behauptet, kein Christ zu sein, versucht - schon durch die Tatsache, daß er Kommunikator ist - zu ermöglichen, wozu Christus gekommen ist.

Aber ich will auch noch ein anderes Beispiel geben, das vielleicht helfen kann, die Aufgabe des Kommunikators zu begreifen: Der christliche Kommunikator von heute ist eine Version oder ein moderner Ausdruck für Prophet. Im Alten Testament gab es eine Institution, die Prophetentum genannt wurde. Dies setzt sich bis heute auf mancherlei Art fort. Die Kommunikatoren sind jedoch auf ganz besondere Weise 
aufgefordert, Propheten zu sein, die Aufgabe der Propheten in der Gesellschaft wahrzunehmen.

Ich will versuchen, in wenigen Worten zu erklären, welche Bedeutung das Prophetentum als Einrichtung im Volk Gottes hatte. Es gab eine Institution der Führerschaft wie das Königstum usw. Es gab auch eine Institution, die das Volk zu Gott hin führen sollte, das Priestertum. Die einzige Schwierigkeit bei diesen Einrichtungen bestand darin, daß sie einfach institutionalisiert, in hohem Maße zur Gewohnheit geworden waren. Mit anderen Worten: sie waren in eine feste Form gepreßt. Die Menschen wußten genau, was sie zu tun hatten. Es gab feste Normen und Methoden und Erwartungen, die an die Menschen, wie auch an die Rolle, die sie in der Gesellschaft zu spielen hatten, gestellt wurden. So waren sie gewissermaßen Sklaven dieses Vorbilds, ihres Bildes in der Gesellschaft. War man König, mußte man in dieser oder jener Weise handeln. Und so handelte man auch. Tat man etwas anderes, sagten die Leute: Wir sind nicht zufrieden mit ihm. Er verhält sich nicht mehr wie ein König. Nicht anders war es bei den Priestern. Sie sollten die Menschen näher zu Gott bringen, besonders im Sinne des Bundes. Aber ein Priester ist eben Priester. Er hat eine bestimmte Funktion in der komplexen Maschinerie der menschlichen Gesellschaft, und man erwartet von ihm, daß er diese Funktion erfüllt. Man erwartet von $i h m, d a ß$ er dieses oder jenes, und es auf diese oder jene Art tut. Die Leute wären erstaunt, wenn er es anders machte. „Wie kommt es, daß die Priester heute so handeln? So verhält sich ein Priester nicht!" Es bedarf dieses Funktionalismus, dieser gewohnheitsmäßigen und institutionalisierten (wenn ich das einmal so sagen darf) Funktionen in der Gesellschaft. Sie sind wichtig. Sie dienen der Gesellschaft. Man schreibt den Priestern bestimmte stereotype Handlungsweisen vor, Rollen, die sie zu spielen haben, weil sie die Gesellschaft festigen. Daher sind die Priester sehr nützlich. Aber es besteht die Gefahr, daß die Dynamik des Fortschritts, die Offenheit für den Fortschritt im Sinne Gottes, möglicherweise erstickt wird, wenn es nur diese festen Institutionen gibt. Der Mensch ist immer in Bewegung. Der Mensch ist immer gerufen, den Plan Gottes zu erfüllen. Er bemüht sich immer darum, dem Rufe Gottes getreuer zu folgen, und dieser Ruf Gottes ist ein sehr freier, unaufdringlicher und unabhängiger Ruf. Er ist Ausdruck der Freiheit Gottes selbst, so daß man zu keinem Zeitpunkt vorhersehen kann, wohin Gott den Menschen führen wird und was er von ihm fordert. Immer "auf dem Sprung sein", immer bereit sein zu hören, den Ruf Gottes zu erkennen, bereit sein, aufzubrechen und ihm zu folgen, wo immer er einen hinführt. Es kann bisweilen sein, daß diese festen Institutionen uns dazu verleiten zu glauben, wir wüßten bereits, wohin uns Gott führt, und wir könnten daher den wirklichen Ruf Gottes überhören. So könnten wir unter Umständen überhören, was er uns heute, jetzt, sagt, weil wir auf ein Sprechen in einer bestimmten Form hören. Daher brauchen wir die Propheten. Propheten sind Menschen, die Vermittler spielen, die für Gott sprechen, in jenen Umständen, in denen die Menschen dahinleben, kämpfen, immer gefordert durch den Bund mit Gott. Sie versuchen, sich diesen Ereignissen, Umständen und Wechselfällen zu stellen, sie zu meistern, aber immer im Blick auf den Bund mit Gott. Und dazu bedarf es des Gewissens. Es bedarf einer „Führung". Die Propheten waren in erster Linie das Gewissen des Volkes. Sie erspürten mit dem Volk und für das Volk, was Gott von ihm verlangte, und erspürten mit dem Volk und für das Volk, wie es Gottes Ruf beantworten und folgen sollte. Sie waren fähig, dies in Worte zu kleiden, denn sie waren Männer des Wortes. Sie waren Spezialisten des Wortes. Sie waren imstande, dies in Worte zu fassen, in eine sinnvolle Rede, so daß Gott mit ihrem Mund zu seinem Volke sprechen konnte. Daher 
nennen wir sie "Sprachrohr" Gottes. Ihre eigene Reaktion, die sie einerseits stellvertretend für die ganze Gesellschaft und andererseits für Gottes Willen hatten, die Art und Weise, wie sie von den Ereignissen betroffen wurden, und die Fähigkeit, die Vision nicht nur in ihrem Inneren zu sehen, sondern sie auch anderen gegenüber wirkungsvoll zum Ausdruck zu bringen, machte sie zu Spezialisten des Wortes. Wenn sie predigten, ließen sie andere an ihrer Vision teilhaben. Sie veranlaßten die Menschen auch, auf Ereignisse zu achten, die an sich banal und farblos erscheinen, die so einen Sinn erhalten. Mit anderen Worten, sie durchbrachen das Banale an den Ereignissen und interpretierten sie im Lichte Gottes. So halfen sie den Menschen, die Dinge des Alltags in einem neuen Licht zu sehen, im Licht der Gegenwart Gottes. Sie zwangen die Menschen, die Ereignisse nicht nur als alltägliche Ereignisse zu sehen, sondern als Aufforderung, den Willen Gottes zu erfüllen. So standen sie immer im Dienste des Volkes und erlaubten ihm nie, müßig auszuruhen und zu sagen: Wir kennen den Willen Gottes. Wir haben Gottes Willen erfüllt. Vielleicht haben wir ihn doch nicht effüllt. Das war ihre Aufgabe: jene Unruhe im Volk zu verbreiten, die die Menschen immer wach hielt in der Erkenntnis, daß sie noch nicht alles getan hätten, das Wort Gottes zu erfüllen.

Die Aufgabe des christlichen Kommunikators besteht nun nicht nur darin, etwas zu sagen. Er muß sich auch selbst mit dem Volk Gottes identifizieren. Volk Gottes bedeutet die Gesamtheit der Menschheit, von Gott gerufen, sein Reich auf Erden zu bauen. Der christliche Kommunikator muß das Gespür haben, eins zu sein mit den Menschen, die sich Gott und seiner Aufforderung stellen, und er muß die Kraft und die Fähigkeit haben, die Ereignisse und Realitäten, denen sie ausgesetzt sind, im Lichte dieses Rufes Gottes zu erklären. Er muß den Menschen gegenüber aufrichtig sein und das darstellen, was wirklich geschieht. Wir Menschen haben Angst, uns selbst gegenüber ehrlich zu sein. Wir täuschen uns gerne über die Dinge hinweg. Wir sagen gerne: Es ist alles in Ordnung. Und: Der andere ist im Unrecht usw. Aber der Prophet stellt den Menschen immer vor dem Angesicht Gottes sich selbst gegenüber. Das sollen auch die Kommunikatoren. Das Volk neigt dazu, Götzen anzubeten. Im Alten Testament dachten die Israeliten: Im neuen Kanaan beten wir keine Götzen an. Seit wir die Wüste verlassen haben, gibt es für uns nur noch einen Weg vorwärts. Wir müssen neue Wege finden, um uns an die neuen Bedingungen in Landwirtschaft und Stadtleben in Kanaan anzupassen. Das war alles gut und schön. Und das möchten auch wir gerne so sagen. Aber dann kamen die Propheten und sagten: Nein, ihr habt das Bündnis vom Sinai gebrochen und lauft Baal usw. nach. So sieht das aus. Ihr habt ein goldenes Kalb geschaffen und betet es an. "Nein, wir beten Jahwe an." "Nein, Ihr betet nicht Jahwe an, sondern das Kalb." Es bedurfte des Propheten, damit sich das Volk seiner eigenen Verantwortung bewußt wurde. Entwirrung schaffen, den Menschen sich selbst offenbaren. So waren die Propheten das Gewissen des Volkes.

Ith bin der Ansicht, daß die erste Forderung, die der christliche Kommunikator erfüllen muß, darin besteht, Gewissen der Menschheit zu sein. Gewissen - nicht auf hochmütige Art. Es gibt eine Art, Prophet zu sein, die falsch ist und hohl klingt. Das ist die Haltung des „Besser-sein-als-der-andere“. Ein Prophet braucht nicht vorzugeben, besser $\mathrm{zu}$ sein als andere. Tatsächlich waren einige unter den Propheten ganz gewöhnliche Menschen. Ich bin der Meinung, daß sie nicht besser waren als andere auch, aber sie wurden zu einem wesentlichen Bestandteil der anderen. Manche der Propheten waren nicht besonders heilige Menschen. In Wirklichkeit hatten die Pro- 
pheten wahrscheinlich die gleichen menschlichen Schwierigkeiten, die gleiche mensdhliche Pilgerschaft durchzumachen, die gleichen Ungewißheiten zu ertragen wie andere auch. Aber sie hatten die Gabe, von Gott Einsichten vermittelt zu bekommen: Einsichten in das menschliche Leben und seinen Sinn, in Ereignisse und ihre Bedeutung. Und das ist der Beitrag, den sie im Namen Gottes leisteten. Es ist nicht etwas, das ihnen naturgemäß eigen ist, sondern eine Gabe, und diese müssen sie zum Wohle der Menschheit nutzen. Es genügt nicht, wenn der Kommunikator die Anforderungen des Lebens eben nur aufzeigt, die Forderungen, die die Wirklichkeit stellt, usw. Er muß auch Glauben inspirieren, Hoffnung wecken, Mut machen, indem er sagt: Das bringen wir zustande!

Der Kommunikator veröffentlicht in den Medien Dinge, die dem Menschen helfen können, sich selbst besser zu verstehen, sein Schicksal in dieser Zeit zu verstehen, den wahren Sinn dessen zu deuten, was in der Welt vor sich geht. Auf diese Art und Weise kann man heute, in unserer modernen Welt, die Funktion des Propheten erfüllen. Doch kann man auch ein falscher Prophet sein. Es gab auch im Volk Gottes Menschen, die falsche Propheten waren. Und weshalb falsch? Einmal weil sie die Wirklichkeit nicht so darstellten, wie sie war, sondern den Leuten nach dem Mund sprachen. Jeremias sagte: Wenn das, was der Prophet weissagt, nicht eintrifft, war es ein falscher Prophet. Wenn es aber eintrifft, war er ein wahrer Prophet. Nun, das ist ganz schön gesagt, aber was meint er damit? Ein Prophet ist ein wahrer Prophet, wenn er sich an die Wirklichkeit hält und nicht phantasiert oder etwas so darstellt, wie die Menschen es hören wollen. Ein Prophet beweist Mut, wenn er die Wirklichkeit über alles andere stellt. Falsche Propheten gab es vor allem an den Königshöfen. Sie wollten dem König gefallen. Sie wollten Dinge sagen, die dem König oder dem Volk gefielen. So sagten sie etwa: „Friede! Friede!“ Als König Achab fragte: „Was passiert im Krieg?“, antwortete der Prophet: „Oh, ich seh den tapferen König, der auf einem Pferd reitet und den Feind in die Flucht schlägt". Und der König fragte verwundert: "Wirklich?" „Mmm, nein.“ König Jehoschaphat fragte: „Haben Sie keinen anderen Propheten?" Adhab: „Ich habe da jemanden, der ist unangenehm, der sagt Schlechtes.“ König Jehoschaphat sagte: „Das macht nichts.“ Der Prophet Michajehu kam. „Du bist ein wahrer Prophet. Kannst Du uns sagen, was passiert?“ Er sagte: „Sie wollen, daß ich weissage wie die anderen." So begann er denn wie die anderen: „Ich sah den König, ich sah den König.“ Der König unterbrach ihn: „Hör auf, wir haben genug davon.“ Er sagte: „Ja, ich sehe den armen König getötet, und seine Schafe in den Bergen zerstreut. ${ }^{“ 2}$ Hier sagt er etwas Unangenehmes, aber das ist das Los der Propheten. Er muß der Wirklichkeit gegenüber treu bleiben und die Dinge nicht aufbauschen, denn er dient niemandem, wenn er nicht die Wahrheit sagt.

Zum zweiten ist der Prophet ein falscher Prophet, wenn er Sklave bestimmter Interessen wird, Sklave von Einzelinteressen, entweder seinen eigenen oder denen bestimmter einzelner in der Gesellschaft. Wenn er sich zum Sklaven und Diener dieser Interessen macht, dann ist er ein falscher Prophet. Da kamen die Propheten und sagten dem Volke: Ja, ihr müßt die fetten Kühe schlachten. Sie wußten nämlich, daß sie von diesen Kühen ihren guten Teil erhalten würden. Sie waren falsche Propheten, weil sie nur auf ihre eigenen Interessen sahen. Oder wenn sie die Interessen einer bestimmten Gesellschaftsschicht vertraten, sei es einer Gruppe von Reichen oder Armen, die jedoch böse Absichten verfolgten. Wann immer sie in der Gesellschaft Partei ergriffen, verloren sie die Freiheit des Geistes Gottes, waren sie keine wahren 
Propheten mehr. Und genauso ist es auch bei den Journalisten, den Kommunikatoren. Wenn sie nur Gott und seiner Wahrheit dienen, dann sind sie Diener der Menschheit. Aber wenn sie Partei ergreifen und irgendwelchen Interessen dienen, dann sind sie als Propheten wertlos, da sie ihre geistige Freiheit verloren haben.

Unsere Aufgabe ist heute zweifach: Vor allem müssen wir wahre Kommunikatoren sein. Wo immer wir herkommen, wo immer wir arbeiten, welcher Glaubensgemeinschaft wir auch angehören, welches auch immer unsere spezielle Aufgabe sein mag, wir sind Kommunikatoren im Dienste des zu errichtenden Reiches Gottes. Je mehr wir lernen, so zusammenzuarbeiten, umso wirksamer wird unsere Arbeit sein. Dies gilt zweitens dann aber auch für das ökumenische Anliegen: Wenn wir wahre Kommunikatoren sind, müssen wir lernen, damit aufzuhören, allein den Interessen unserer kleinen Kirche zu dienen. Wir müssen erkennen, daß unsere Sendung den Rahmen einer Institution übersteigt. Der Beruf des Kommunikators ist größer, als daß er nur einer kleinen Sache diente. Er dient Gott, der seinen Ruf an die ganze Welt richtet. Die Journalisten sollten uns lehren, von subjektiver Parteinahme frei zu einer Sicht zu gelangen, die umfassend ist wie der Ruf Gottes. Es ist gut, so auch mit Menschen aus verschiedenen Glaubensgemeinschaften zusammenzutreffen und $z u$ lernen, eins zu sein, und sich nicht durch kleine Unterschiede in der gemeinsamen Aufgabe binden oder trennen zu lassen.

Anmerkungen:

1. Vgl. Communio et Progressio 10,4.

2. Vgl. 1 Kön 22.

Being a communicator is already a very sacred job. God created men as a social being and society cannot be without mutual information, without communication. Thus, the communicator is standing right in the heart of human existence. In fulfilling his task, he is helping in God's plan towards perfection of the world, a plan, which, according to God's will, can only be accomplished through mutual giving and receiving. The picture of the Communicator in the Bible is double: the tower of Babel, where men try to build up a world without God, but consequently, breaking down communication, and Pentecost, where all men understood the message of the Spirit despite the different languages. Christ becoming man became God's communication with and for us. In him, all communication is highlighted. Also the Old Testament prophet is example for the communicator. He was not determinated by a defined function in society, but was bound only towards the Word of God. The prophets were God's conscience with the people like any modern communicator has to be today. It is the duty of the christian communicator to be at this service for the establishment of God's Kingdom. 


\section{RÉSUME}

Déjà, le fait en soi d'être communicateur est une très haute tâche. Dicu a créé l'homme en tant qu'être communicateur, et il n'y a pas de communauté possible sans relation avec autrui, sans communication. Ainsi, le communicateur se trouve au centre de l'être humain. En accomplissant une telle tâche, il coopère au plan de Dieu en vue de la parfaite exécution du monde qui, selon sa volonté, ne peut être atteinte que par un donner et un recevoir mutuels. L'image du communicateur, selon l'Ecriture Sainte, est double: la Tour de Babel où les hommes essaient de construire un monde sans Dieu et ainsi s'effondre la communication; la Pentecôte où tous les hommes comprennent l'unique langue de l'Esprit et où la communication a lieu par-delà les différentes langues. L'Incarnation fut la communication de Dieu avec et pour nous. En lui se résume chaque communication. De même, l'aspect du prophète de l'Ancien Testament est le modèle du communicateur. Il n'était pas contraint à une fonction définie dans la société, mais seulement l'obligé de la voix de Dieu. Les prophètes étaient la conscience du peuple, de même que le communicateur doit l'être aussi de nos jours. C'est le devoir du communicateur chrétien de se mettre au service de l'établissement du royaume de Dieu.

\section{R E S U M E N}

Ya el solo hecho de ser-comunicante constituye un cometido importante. Dios ha creado al hombre como ser social y la sociedad no es posible sin comunicación mútua. Por ello el comunicante se encuentra en lugar central de la naturaleza humana. Con el desempeño de este cometido coopera en el plan de Dios para lograr la perfección del mundo, que, según la voluntad divina, sólo es posible mediante un dar y recibir recíproco. La imagen de la comunicación en la Sagrada Escritura es doble: la construcción de la torre de Babel, donde los hombres tratan de crear un mundo sin Dios, empeño que se desploma con la falta de comunicación; en segundo lugar Pentecostés, donde todos los hombres comprenden el lenguaje único del Espíritu y se da una comunicación que supera las barreras de las lenguas diversas. La encarnación de Cristo fué comunicación de Dios con y para nosotros. En Ell se halla contenida toda comunicación. Incluso la figura del profeta veterotestamentario es prototipo del comunicador. No se encuentra coartada por el desempeño de una función social determinada sino obligada únicamente por la voz de Dios. Los profetas eran la conciencia del pueblo al igual que el comunicador de hoy. La misión de éste, en cuanto cristiano, es estar al servicio del logro del reino de Dios. 